\title{
Analysis of Curative Effect and Influencing Factors of NI Stage Papillary Thyroid Micro-Carcinoma and Papillary Thyroid Non-Micro Carcinoma After Initial Radioactive lodine Ablation Therapy
}

This article was published in the following Dove Press journal:

Cancer Management and Research

\author{
Canhua Yun (1D) \\ Meiling $\mathrm{Wu}^{\prime}$ \\ Juan $\mathrm{Xiao}^{2}$ \\ Yong Liu' \\ Wei Zhang' \\ Jingjia Cao $\mathbb{D}^{\prime}$ \\ 'Department of Nuclear Medicine, The \\ Second Hospital, Cheeloo College of \\ Medicine, Shandong University, Jinan, \\ 250033, People's Republic of China; \\ ${ }^{2}$ Center of Evidence-Based Medicine, \\ Institute of Medical Sciences, The Second \\ Hospital, Cheeloo College of Medicine, \\ Shandong University, Jinan, 250033, \\ People's Republic of China
}

Correspondence: Jingjia Cao

No. 247, Beiyuan Street, Jinan City, Shandong Province, People's Republic of China

Tel +86 17660083825

Email sdhyx1990@163.com
Objective: To compare the efficacy and influencing factors of initial radioactive iodine (RAI) ablation therapy for postoperative N1 stage papillary thyroid micro-carcinoma (PTMC) and papillary thyroid non-micro carcinoma (PTC), and to explore the necessity of RAI for N1 stage PTMC.

Methods: A retrospective analysis of patients with N1 stage papillary thyroid cancer who underwent RAI in our department from January 2018 to June 2019. According to the tumor diameter, papillary thyroid carcinoma was divided into PTMC group $(\leq 1.0 \mathrm{~cm})$ with 129 patients and PTC group $(>1.0 \mathrm{~cm}$ ) with 214 patients. According to the 2015 ATA guidelines, the patient's treatment response was evaluated 6-8 months after discharge from the hospital: excellent response (ER), indeterminate response (IDR), biochemical incomplete response (BIR), and structural incomplete response (SIR). IDR, BIR, and SIR were classified into NER group. Chisquared test, independent sample $t$-test, Mann-Whitney $U$ test, and binary logistic regression analysis were used to compare the differences between PTMC and PTC patients.

Results: The ps-Tg of the PTMC group was significantly lower than that of the PTC group $(P=0.001)$, and the ER ratio of the PTMC group was higher $\left(\chi^{2}=5.445, P<0.05\right)$. The ER ratio of PTMC patients in the N1a group was significantly higher than that of PTC patients $\left(80 \%, 66.7 \%, \chi^{2}=4.076, P<0.05\right)$, while the ER ratio of PTMC in the N1b group was not significantly different from that of PTC. Gender, N stage, and ps-Tg were found to be independent factors of RAI treatment response.

Conclusion: The efficacy of the initial RAI of PTMC patients was significantly better than that of PTC patients. There was no significant difference in the efficacy of RAI between males with PTMC, N1b stage, ps-Tg $\geq 5.87 \mathrm{ng} / \mathrm{mL}$ and PTC patients, which suggested that RAI is necessary for these patients.

Keywords: papillary thyroid micro-carcinoma, papillary thyroid non-micro carcinoma, curative effect, RAI

\section{Background}

Recently, the incidence rate of papillary thyroid carcinoma has been increasing. Among it, the papillary thyroid micro-carcinoma (PTMC) is the fastest growing type. However, its diagnosis and treatment remain controversial. ${ }^{1}$ Radioactive iodine (RAI) ablation is an important adjuvant therapy for papillary thyroid carcinoma patients postoperation. It can significantly reduce the risk of recurrence and 
death of papillary thyroid carcinoma patients. ${ }^{2}$ Compared with papillary thyroid non-micro carcinoma (PTC), PTMC lesions are more limited and relatively inactive, and have better clinical prognosis. However, PTMC is still aggressive and can cause local recurrence and cervical lymph node metastasis. PTMC is not equivalent to low-risk cancer. $^{3}$ At present, it is considered that RAI treatment is not necessary for PTMC in $\mathrm{N}_{0} \mathrm{M}_{0}$ stage. But there is still disagreement about whether patients with N1a-N1b stage need RAI treatment or not. ${ }^{2,4}$ According to the 2015 edition of the American Thyroid Association (ATA) guidelines, the clinical outcomes of patients after initial RAI can be divided into excellent response (ER), indeterminate response (IDR), biochemical incomplete response (BIR), and structural incomplete response (SIR). ${ }^{4}$ The recurrence rate of ER patients is only $1 \%-4 \%$ and the risk of diseasespecific death is less than $1 \% .15 \%-20 \%$ of IDR patients will have structural disease identified during follow-ups. The probability of it developing into structural disease in BIR patients is about $20 \%$. However, $50 \%-85 \%$ of patients continue to have persistent disease with SIR despite additional therapy. For patients with SIR, the risk of disease-specific death rates was as high as $11 \%$ with loco-regional metastases and structurally distant metastases which were found in $50 \%$ of patients. ${ }^{4}$ At present, few studies compared the therapeutic efficacy of RAI between PTC and PTMC. The aim of this study was to compare the efficacy of RAI between PTC and PTMC in stage N1 through retrospectively analyzing the data in our hospital.

\section{Data and Methods}

\section{Research Population}

Patient data extracted from the clinical electronic medical record system were de-identified so that all private information of patients was not included. The Institutional Review Board of the Second Hospital, Cheeloo College of Medicine, Shandong University approved the use of medical records and allowed us to obtain written consent from each patient (KYLL-2018[LW]013). All procedures complied with the Declaration of Helsinki for research involving human subjects. All the patients signed informed consent before RAI.

The data of patients with papillary thyroid carcinoma treated with initial RAI were retrospectively collected from January 2018 to June 2019. Patients with total thyroidectomy/near-total thyroidectomy combined with lymph node dissection were included. The patients with one of the following: incomplete clinical data; inadequate information of follow-up; no cervical lymph node metastasis; distant metastasis (examined by ultrasonography, chest CT, 131I whole body imaging, whole body bone imaging or magnetic resonance imaging, etc.); PTMC with only one tumor lesion; bad subtypes (such as: tall cell variant, diffuse sclerosing variant, columnar cell variant, etc.); positive anti-thyroglobulin antibody $(\mathrm{TgAb})>4000 \mathrm{U} / \mathrm{L})$ or preablative-stimulated TSH (ps-TSH) $<30 \mathrm{uIU} / \mathrm{mL}$ were excluded.

\section{Grouping and Information of Collected Data}

According to the maximum postoperative tumor diameter, the patients were divided into PTC group (tumor diameter $>1.0 \mathrm{~cm}$ ) and PTMC group (tumor diameter $\leq 1.0 \mathrm{~cm}$ ). According to the eighth edition of AJCC, patients were divided into N1a and N1b stage.

The data including gender, age, pathological information (extrathyroidal extension (ETE), Hashimoto's thyroiditis (HT), single and double lobes), treatment time (TT) from postoperation to the initial RAI treatment, urinary iodine level, ps-TSH, preablative-stimulated thyroglobulin (ps-Tg), lymph node metastasis, and other data were collected.

\section{Initial RAI Therapy}

Before admission, the patients were ordered to stop taking levothyroxine sodium tablets and have a low iodine diet for 4 weeks. The implementation was self-managed by the patients. On the day of admission, fasting urine and blood samples of patients were collected. Urinary iodine level and serological indexes (ps-Tg, TgAb, ps-TSH, free triiodothyronine, free thyroxine, triiodothyronine, thyroxine) were recorded. If no contraindications, all patients included had ablation with $3.70 \mathrm{GBq}(100 \mathrm{mCi}) 131 \mathrm{I}$ administered orally. A whole body post-treatment scan (Rx-131I-WBS) was performed 3 days after RAI treatment. 6-8 months after discharge, patients were monitored for serological indicators ( $\mathrm{Tg}, \mathrm{TgAb}, \mathrm{TSH}$, free triiodothyronine, free thyroxine, triiodothyronine, and thyroxine), neck ultrasonography, and diagnostic whole body 131I imaging (Dx-131I-WBS).

\section{Laboratory Detection Methods}

Urinary iodine level was determined by arsenic-cerium catalytic spectrophotometry with mild acid digestion, and the determination range was $30-1500 \mathrm{ug} / \mathrm{L}$. Tg and $\mathrm{TgAb}$ 
were determined by electrochemiluminescence immunoassay in the range of $0-464 \mathrm{ng} / \mathrm{mL}$ and $0-4000 \mathrm{U} / \mathrm{L}$, respectively. TSH was determined by the chemiluminescence method in the range of $0-100.0 \mathrm{uIU} / \mathrm{mL}$. Ps-TSH $>100$ $\mathrm{uIU} / \mathrm{mL}$ was recorded as $100 \mathrm{uIU} / \mathrm{mL}$.

\section{Definitions of Response to RAI}

Based on imaging findings and stimulated/suppressed $\mathrm{Tg}$ levels of the initial follow-up examination, patients were classified into four response-to-therapy categories recommended by the ATA guidelines as shown in Table 1 . According to ATA guidelines, considering follow-up Tg level, Dx-WBS examination, neck ultrasonography, and other imaging examinations, patients were divided into two groups: ER group and NER group. IDR, BIR, and SIR were classified into NER group. Three nuclear medicine physicians independently assessed the outcome of follow-up examinations after treatment and reached agreement through consultation when discordance appeared.

\section{Statistical Analysis}

SPSS 20.0 statistical software was used for analysis. The categorical data were described as cases and percentages. Continuous data with normal distribution were expressed as means \pm standard deviation (SD). Continuous data with skewed distribution were expressed as median (quartile). $\chi^{2}$ test, independent sample $t$-test, and Mann-Whitney $U$ test were used to compare the differences between two groups of patients. The binary logistic regression model was established, and the factors with $P<0.10$ in univariate analysis were included in the model to further explore the independent factors affecting the prognosis. In addition, receiver operating characteristic (ROC) curve was performed to determine the cut-off value of ps-Tg for ER. $P<0.05$ was considered statistically significant.

\section{Result \\ Basic Characteristics of Patients in PTC and PTMC Groups}

129 PTMC patients (38 males and 91 females, mean age $42.78 \pm 10.52$ years) and 214 PTC patients (61 males and 153 females, mean age $41.71 \pm 11.75$ years) were included. There were no significant differences in gender, age, presence or absence of ETE, HT, single and double lobes, TT, urinary iodine level, ps-TSH between the two groups (all $P>0.05)$. The ps-Tg of the PTMC group was significantly lower than that of the PTC group, and the difference was statistically significant $(P=0.001)$. After the patients were further divided into N1a and N1b groups, the ps-Tg between the two groups was also significantly different $(P$ values were 0.001 and 0.006 , respectively) (Table 2).

\section{Relationship Between Lymph Node Metastasis and Treatment Response in PTC and PTMC Groups}

Patients were divided into ER group and NER group according to the response after RAI. The ER ratio of the PTMC group was significantly higher than that of the PTC group $(P=0.02)$. After the patients were further divided into N1a and N1b groups, the ER ratio of the PTMC group in the N1a group was significantly higher than that of the PTC group, while the ER ratio of the PTMC group in the N1b group was not significantly different compared with that of PTC group (Table 2).

Comparing the number of lymph node metastases and response to RAI between the N1a group and the N1b

Table I Clinical Implications of Response to Therapy Reclassification in Patients with Differentiated Thyroid Cancer Treated with Total Thyroidectomy and Radioiodine Remnant Ablation

\begin{tabular}{|l|l|}
\hline Category & Definitions \\
\hline Excellent response (ER) & Negative imaging and either suppressed $\mathrm{Tg}<0.2 \mathrm{ng} / \mathrm{mL}$ or stimulated $\mathrm{Tg}<\mathrm{I} \mathrm{ng} / \mathrm{mL}$ \\
\hline $\begin{array}{l}\text { Biochemical incomplete } \\
\text { response (BIR) }\end{array}$ & Negative imaging and suppressed $\mathrm{Tg} \geq \mathrm{I} \mathrm{ng} / \mathrm{mL}$ or stimulated $\mathrm{Tg} \geq 10 \mathrm{ng} / \mathrm{mL}$ or rising anti-Tg antibody levels \\
\hline $\begin{array}{l}\text { Structural incomplete } \\
\text { response (SIR) }\end{array}$ & Structural of functional evidence of disease with any $\mathrm{Tg}$ level with or without anti- $\mathrm{Tg}$ antibodies \\
\hline Indeterminate response (IDR) & $\begin{array}{l}\text { Non-specific findings on imaging studies; faint uptake in thyroid bed on RAl scanning; non-stimulated Tg } \\
\text { detectable, but }<\mathrm{I} \mathrm{ng/mL;} \mathrm{stimulated} \mathrm{Tg} \mathrm{detectable,} \mathrm{but}<10 \mathrm{ng} / \mathrm{mL} \text { or anti-Tg antibodies stable or declining in the } \\
\text { absence of structural or functional disease }\end{array}$ \\
\hline
\end{tabular}


Table 2 General Data of PTC and PTMC and the Relationship Between Lymph Node Metastasis and Treatment Response

\begin{tabular}{|c|c|c|c|c|}
\hline & PTC $(n=214)$ & PTMC (n = I29) & $\chi^{2}$ & $P$ \\
\hline Male (case) & 61 (28.5\%) & 38 (29.5\%) & 0.036 & 0.85 \\
\hline Female (case) & I53 (7I.5\%) & 91 (70.5\%) & & \\
\hline Age (years) & $41.7 I \pm I 1.75$ & $42.78 \pm 10.52$ & & 0.401 \\
\hline$\geq 55$ (case) & 25 (II.7\%) & $20(15.5 \%)$ & 1.031 & 0.31 \\
\hline < 55 (case) & 189 (88.3\%) & 109 (84.5\%) & & \\
\hline ETE (case) & 47 (22\%) & $22(17.1 \%)$ & 1.207 & 0.272 \\
\hline No ETE (case) & 167 (78\%) & 107 (82.9\%) & & \\
\hline HT (case) & $23(10.7 \%)$ & $12(9.3 \%)$ & 0.183 & 0.668 \\
\hline No HT (case) & 191 (89.3\%) & 117 (90.7\%) & & \\
\hline Single lobe (case) & 115 (53.7\%) & $69(53.5 \%)$ & 0.002 & 0.964 \\
\hline Double lobe (case) & 99 (46.3\%) & $60(46.5 \%)$ & & \\
\hline TT (day) & 7I $(58,89)$ & $77(62.5,93)$ & & 0.06 \\
\hline Urinary iodine level (ug/L) & $103(59.8,195.4)$ & $95.5(64.9,167.5)$ & & 0.44 \\
\hline ps-TSH (ulU/mL) & $98.91(72.88,100.00)$ & $98.30(71.46,100.00)$ & & 0.9 \\
\hline $\mathrm{ps}-\mathrm{Tg}(\mathrm{ng} / \mathrm{mL})$ & $7.67(4.8,16.97)$ & $3.51(1.36,10.12)$ & & 0.001 \\
\hline Nla ps-Tg (ng/mL) & $7.39(4.75,13.57)$ & $3.19(1.22,9.54)$ & & 0.001 \\
\hline NIb ps-Tg (ng/mL) & $9.09(4.95,23.22)$ & $4.39(1.88,14.38)$ & & 0.006 \\
\hline Total ER (case) & 117 (54.7\%) & 87 (67.4\%) & 5.445 & 0.02 \\
\hline Total NER (case) & 97 (45.3\%) & $42(32.6 \%)$ & & \\
\hline NIa ER (case) & $68(66.7 \%)$ & $58(80.6 \%)$ & 4.076 & 0.044 \\
\hline NIa NER (case) & $34(33.3 \%)$ & 14 (19.4\%) & & \\
\hline Nla Lymph node metastasis $\geq 3$ ER (case) & $20(54.1 \%)$ & $14(70 \%)$ & 1.372 & 0.242 \\
\hline Nla Lymph node metastasis $\geq 3$ NER (case) & $17(45.9 \%)$ & $6(30 \%)$ & & \\
\hline NIb ER (case) & $49(43.8 \%)$ & $29(50.9 \%)$ & 0.772 & 0.38 \\
\hline NIb NER (case) & $63(56.2 \%)$ & $28(49.1 \%)$ & & \\
\hline NIb Lymph node metastasis $\geq 3$ ER (case) & $24(31.6 \%)$ & $16(38.1 \%)$ & 0.513 & 0.474 \\
\hline NIb Lymph node metastasis $\geq 3$ NER (case) & $52(68.4 \%)$ & $26(61.9 \%)$ & & \\
\hline
\end{tabular}

Abbreviations: PTC, papillary thyroid non-micro carcinoma; PTMC, papillary thyroid micro-carcinoma; ps-TSH, preablative-stimulated TSH; ps-Tg, preablative-stimulated thyroglobulin.

group, no significant difference in treatment response was found between the PTC group and the PTMC group $(P>$ 0.05). However, with the stage from N1a to N1b and the number of lymph node metastases increasing, the percentage of ER gradually decreased and the percentage of NER gradually increased (Figure 1).

\section{Comparison of PTC and PTMC According to RAI Response (ER and NER)}

In the PTC group, female gender, single lobe, and N1a were protective factors for treatment response, and ps-Tg in the ER group was significantly lower than that in the
NER group $(P<0.05)$. The median ps-Tg of the ER group was $5.39 \mathrm{ng} / \mathrm{mL}(4.33,8.84)$, which was significantly lower than that of the NER group (15.46 ng/mL (7.67, 34.68), $(P=0.001)$ ) (Table 3$)$. Gender, single and double lobes, $\mathrm{N}$ stage, and ps-Tg level were further included into the binary logistic regression model (NER group was regarded as the reference group). The result showed that gender, $\mathrm{N}$ stage, and ps-Tg were independent factors for treatment response (Table 4). The ROC curve of the relationship between ps-Tg and ER is shown in Figure 2. The area under the curve (AUC) was 0.806 and the cut-off value was $\mathrm{ps}-\mathrm{Tg}=10.61$. The sensitivity and specificity 


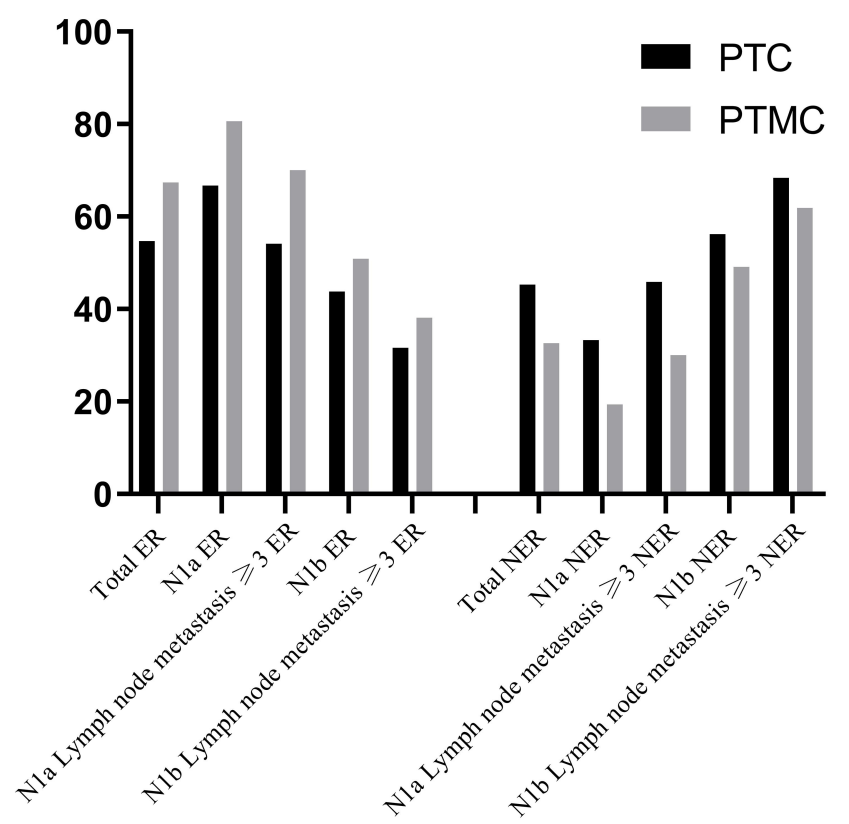

Figure I With the stage from $\mathrm{Nla}$ to $\mathrm{NIb}$ and the number of lymph node metastases increasing, the percentage of ER gradually decreased and the percentage of NER gradually increased.

were 0.753 and 0.744 , respectively, which suggested that the model had good performance.

In the PTMC group, female gender, maximum diameter $<0.5 \mathrm{~cm}$ tumor, and N1a stage were protective factors for treatment response. Ps-Tg in ER group was significantly lower than that in the NER group $(P<0.05)$ (Table 3$)$. Gender, tumor maximum diameter, $\mathrm{N}$ stage, and ps-Tg level were further included in the binary logistic regression model (NER group was regarded as the reference group), and the results showed that gender, $\mathrm{N}$ stage, and ps-Tg were independent factors for RAI treatment response (Table 4). The median ps-Tg of ER group was $3.1 \mathrm{ng} / \mathrm{mL}(1.21,9.7)$, which was significantly lower than that of the NER group, $4.275 \mathrm{ng} / \mathrm{mL}(2.28,17.07)(P=0.007)$. The ROC curve of the relationship between ps-Tg and ER was shown in Figure 2. The AUC was 0.690, the cut-off value was ps$\mathrm{Tg}=5.87$. The sensitivity and specificity were 0.452 and 0.831 , respectively. Using ps-Tg to predict the RAI treatment response, the effect in PTMC was not as good as that in the PTC group.

\section{Discussion}

The incidence rate of thyroid cancer is increasing year by year. Studies have reported that PTMC accounts for nearly $50 \%$ of newly found papillary thyroid carcinoma. ${ }^{5}$ Although PTMC progresses slowly and has a good prognosis, ${ }^{6}$ there is still a local recurrence rate of $2 \%-6 \%$, and distant metastasis occurs in $1 \%-2 \%$ of patients. ${ }^{7}$ At present, the best treatment for papillary thyroid carcinoma is surgery $+\mathrm{RAI}+\mathrm{TSH}$ suppression therapy. RAI can remove the residual thyroid tissue for papillary thyroid carcinoma patients postoperation and clear metastatic lesions, which can effectively reduce the recurrence rate. ${ }^{2}$ Chinese experts reached consensus on diagnosis and treatment of papillary thyroid carcinoma (2016 Edition): for PTMC patients with metastasis (especially distant metastasis), RAI can help to eliminate residual lesions or metastases that cannot be removed by surgery, which aids to alleviate the disease and reduce the risk of recurrence of PTMC. ${ }^{1}$ However, PTMC may have different degrees of cervical lymph node metastasis. AJCC 8th Edition of TNM stage changed the VII area of lymph node metastasis from lateral lymph node metastasis (N1b) to central lymph node metastasis (N1a). ${ }^{8}$ Therefore, in order to alleviate the condition, reduce the recurrence rate, and the possibility of distant metastasis, and to facilitate postoperative $\mathrm{Tg}$ stratification, re-staging and follow-up, increase the sensitivity of early diagnosis of recurrence, and reduce the patient's psychological and medical cost burden, it is of great significance to compare the efficacy and influencing factors of initial RAI between the PTMC group and the PTC group and to explore the necessity of RAI for PTMC in N1 stage.

In this study, $67.4 \%$ of patients in thePTMC group achieved ER status, which was significantly higher than that in the PTC group with $54.7 \%$. The result was consistent with that of Feng et al. ${ }^{3}$ The ps-Tg of the PTC group was significantly higher than that of the PTMC group. After being further divided into N1a group and N1b group, the ER ratio of PTMC in the N1a group was significantly higher than that of the PTC group, and ps$\mathrm{Tg}$ in the N1b group was also significantly higher than that in the N1a group, while the ER ratio of PTMC patients in the N1b group was not significantly different from that in the PTC group. In the further multivariate analysis, $\mathrm{N}$ stage was also an independent influencing factor of RAI treatment response. Compared with the N1a group, the OR value of PTMC in the N1b group was 4.169, which was significantly higher than the 2.197 of PTC. This suggests the necessity of RAI for PTMC in the N1b group compared with the N1a group. Comparing the number of lymph node metastases $(\geq 3)$ in the N1a group and $\mathrm{N} 1 \mathrm{~b}$ group, there was no significant difference in RAI treatment response between the PTC group and the PTMC group. The percentages of ER and NER in each group of N1a, N1a, and lymph node metastasis $\geq 3, \mathrm{~N} 1 \mathrm{~b}$, 
Table 3 Comparison of PTC and PTMC After Being Divided into ER and NER Groups

\begin{tabular}{|c|c|c|c|c|c|c|c|c|}
\hline & ER (PTC) & NER (PTC) & $\chi^{2}$ & $P$ & ER (PTMC) & NER (PTMC) & $\chi^{2}$ & $P$ \\
\hline $\begin{array}{l}\text { Male (case) } \\
\text { Female (case) }\end{array}$ & $\begin{array}{l}22(36.1 \%) \\
95(62.1 \%)\end{array}$ & $\begin{array}{l}39(63.9 \%) \\
58(37.9 \%)\end{array}$ & 11.92 & 0.001 & $\begin{array}{l}18(47.4 \%) \\
69(75.8 \%)\end{array}$ & $\begin{array}{l}20(52.6 \%) \\
22(24.2 \%)\end{array}$ & 9.885 & 0.002 \\
\hline $\begin{array}{l}\text { Age (years) } \\
\geq 55 \text { (case) } \\
<55 \text { (case) }\end{array}$ & $\begin{array}{l}41.7 \pm 11.54 \\
13(52 \%) \\
104(55 \%)\end{array}$ & $\begin{array}{l}41.73 \pm 12.05 \\
12(48 \%) \\
85(45 \%)\end{array}$ & 0.082 & $\begin{array}{l}0.985 \\
0.775\end{array}$ & $\begin{array}{l}43.48 \pm 10.61 \\
16(80 \%) \\
71(65.1 \%)\end{array}$ & $\begin{array}{l}41.31 \pm 10.29 \\
4(20 \%) \\
38(34.9 \%)\end{array}$ & 1.7 & $\begin{array}{l}0.273 \\
0.192\end{array}$ \\
\hline $\mathrm{TT}$ (day) & $74(59,90.75)$ & $66(57,88)$ & & 0.143 & $75(62,95)$ & $79(66.75,88)$ & & 0.962 \\
\hline $\begin{array}{l}>90 \text { days } \\
\leq 90 \text { days }\end{array}$ & $\begin{array}{l}29(64.4 \%) \\
88(52.1 \%)\end{array}$ & $\begin{array}{l}16(35.6 \%) \\
81(47.9 \%)\end{array}$ & 2.196 & 0.138 & $\begin{array}{l}26(72.2 \%) \\
61(65.6 \%)\end{array}$ & $\begin{array}{l}10(27.8 \%) \\
32(34.4 \%)\end{array}$ & 0.52 & 0.471 \\
\hline Urinary iodine level (ug/L) & $106(70.7,200.7)$ & $97.2(57.55,175.8)$ & & 0.154 & $95.4(64,178.2)$ & $103.5(66.7,166.75)$ & & 0.642 \\
\hline ps-TSH (ulU/mL) & $97.34(67.41,100)$ & $99.4(73.25,100)$ & & 0.725 & $98.01(71.88,100)$ & $100(69.99,100)$ & & 0.934 \\
\hline $\mathrm{ps}-\mathrm{Tg}(\mathrm{ng} / \mathrm{mL})$ & $5.39(4.33,8.84)$ & $15.46(7.67,34.68)$ & & 0.001 & $3.1(1.21,9.7)$ & $4.275(2.28,17.07)$ & & 0.007 \\
\hline Maximum diameter $(\mathrm{cm})$ & $1.5(1.2,2.0)$ & $1.5(1.2,2.45)$ & & 0.251 & $0.5(0.5,0.8)$ & $0.7(0.5,0.8)$ & & 0.206 \\
\hline $\begin{array}{l}\text { PTC }>2 \mathrm{~cm} \text { (case) } \\
\text { PTMC }>0.5 \mathrm{~cm} \text { (case) } \\
\text { PTC } \leq 2 \mathrm{~cm} \text { (case) } \\
\text { PTMC } \leq 0.5 \mathrm{~cm} \text { (case) }\end{array}$ & $\begin{array}{l}25(45.5 \%) \\
92(57.9 \%)\end{array}$ & $\begin{array}{l}30(54.5 \%) \\
67(42.1 \%)\end{array}$ & 2.538 & 0.111 & $\begin{array}{l}4 \mathrm{I}(59.4 \%) \\
46(76.7 \%)\end{array}$ & $\begin{array}{l}28(40.6 \%) \\
14(23.3 \%)\end{array}$ & 4.347 & 0.037 \\
\hline $\begin{array}{l}\text { ETE (case) } \\
\text { No ETE (case) }\end{array}$ & $\begin{array}{l}26(55.3 \%) \\
91(54.5 \%)\end{array}$ & $\begin{array}{l}21 \text { (44.7\%) } \\
76(45.5 \%)\end{array}$ & 0.01 & 0.92 & $\begin{array}{l}12(54.5 \%) \\
75(70.1 \%)\end{array}$ & $\begin{array}{l}10(45.5 \%) \\
32(29.9 \%)\end{array}$ & 2.009 & 0.156 \\
\hline $\begin{array}{l}\text { HT (case) } \\
\text { No HT (case) }\end{array}$ & $\begin{array}{l}10(43.5 \%) \\
107(56 \%)\end{array}$ & $\begin{array}{l}13(56.5 \%) \\
84(44 \%)\end{array}$ & 1.303 & 0.254 & $\begin{array}{l}8(66.7 \%) \\
79(67.5 \%)\end{array}$ & $\begin{array}{l}4(33.3 \%) \\
38(32.5 \%)\end{array}$ & & 0.952 \\
\hline $\begin{array}{l}\text { Single lobe (case) } \\
\text { Double lobe (case) }\end{array}$ & $\begin{array}{l}70(60.9 \%) \\
47(47.5 \%)\end{array}$ & $\begin{array}{l}45(39.1 \%) \\
52(52.5 \%)\end{array}$ & 3.852 & 0.05 & $\begin{array}{l}44(63.8 \%) \\
43(71.7 \%)\end{array}$ & $\begin{array}{l}25(36.2 \%) \\
17(28.3 \%)\end{array}$ & 0.912 & 0.34 \\
\hline $\begin{array}{l}\text { Nla (case) } \\
\text { NIb (case) }\end{array}$ & $\begin{array}{l}68(66.7 \%) \\
49(43.8 \%)\end{array}$ & $\begin{array}{l}34(33.3 \%) \\
63(56.2 \%)\end{array}$ & 11.31 & 0.001 & $\begin{array}{l}58(80.6 \%) \\
29(50.9 \%)\end{array}$ & $\begin{array}{l}14(19.4 \%) \\
28(49.1 \%)\end{array}$ & 12.76 & 0.001 \\
\hline
\end{tabular}

$\mathrm{N} 1 \mathrm{~b}$, and lymph node metastasis $\geq 3$ were calculated. It was found that with the stage from N1a to N1b and the number of lymph node metastases increasing, the percentage of ER gradually decreased and the percentage of NER gradually increased. This implies that PTMC may be an

Table 4 Multivariate Logistic Regression Analysis on the RAI Treatment Response in PTC and PTMC

\begin{tabular}{|l|l|l|l|}
\hline & $\boldsymbol{\beta}$ & OR (95\% CI) & $\boldsymbol{P}$ \\
\hline PTC & & & \\
Gender (male) & 0.922 & $2.515(\mathrm{I} .248,5.068)$ & 0.01 \\
N (NIb) & 0.787 & $2.197(1.151,4.194)$ & 0.017 \\
Ps-Tg & 0.095 & $1.099(1.062,1.138)$ & 0.001 \\
\hline PTMC & & & \\
Gender (male) & 1.274 & $3.575(1.483,8.617)$ & 0.005 \\
N (NIb) & 1.428 & $4.169(1.779,9.766)$ & 0.001 \\
Ps-Tg & 0.029 & $1.029(1.000,1.059)$ & 0.049 \\
\hline
\end{tabular}

early lesion of PTC, ${ }^{9}$ it also reflects that the central lymph node is the cervical lymph node metastasis pathway. ${ }^{10}$

In this study, the male to female ratios of PTMC and PTC were 1:2.39 and 1:2.51, respectively. Gender was an independent factor of RAI response in multivariate analysis, which was consistent with the results of Long et al, ${ }^{11}$ indicating that both PTMC and PTC are more likely to occur in female patients. Regarding age ( $\geq 55$ years old) as an independent factor affecting TNM staging, there was no statistical difference in this study $(P>0.05)$. This may be related to the selection of stage $\mathrm{N} 1$ and fewer patients $\geq 55$ years old in this study. As one of the factors that affect RAI treatment response, TT was inconsistent in previous literature. $^{12,13} \mathrm{Li}$ et al considered that among the low and medium risk patients postoperation for differentiated thyroid carcinoma (DTC), the risk of poor prognosis for patients who delayed RAI treatment for more than 3 months was 3.77 times higher than that for patients who 


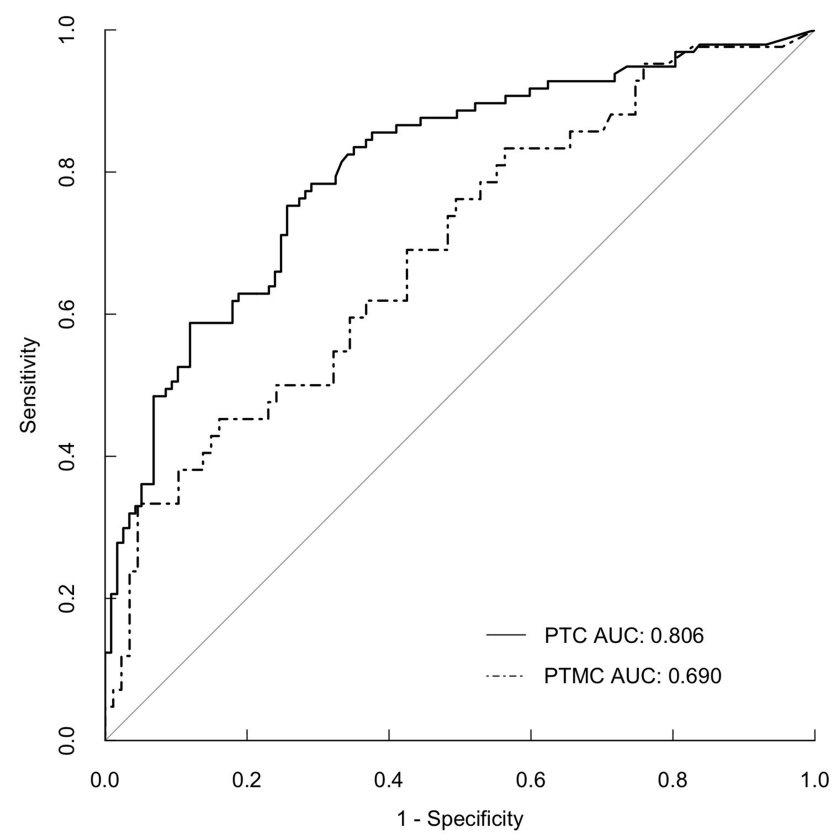

Figure 2 ROC curve of the relationship between ps-Tg and RAI response in patients with PTC and PTMC.

received RAI treatment within 3 months. ${ }^{12}$ Wang et al suggested that RAI treatment within 6 months postoperation may have no significant effect on prognosis. ${ }^{13}$ In this study, the median TT of ER and NER in the PTMC group were 75 days and 79 days, respectively, and the median TT of ER and NER in the PTC group were 74 days and 66 days, respectively, which had no statistical difference. It indicated that TT around 3 months had no significant effect on the efficacy of RAI in PTMC and PTC patients.

TSH can stimulate the expression of NIS on the cell membrane to enhance the 131I absorbed by residual thyroid tissue or DTC lesions, thereby its increase can improve the therapeutic efficacy of RAI. Ps-TSH $>30 \mathrm{uIU} / \mathrm{mL}$ is required for RAI therapy, which was recommended by the ATA guideline. Xin et al believe that for patients with ps-TSH of $90 \sim 120 \mathrm{uIU} / \mathrm{mL}$, the efficacy of RAI is significantly higher than for those with other ps-TSH levels. ${ }^{14}$ However, no statistical difference of ps-TSH levels was found between the ER and NER groups among PTC and PTMC patients in this study, which may be associated with the fact that the upper limit of ps-TSH was $100 \mathrm{uIU} / \mathrm{mL}$ in this study. It is widely agreed that ps-Tg levels influence RAI response. ${ }^{15,16} \mathrm{Kim}$ et al considered that the ps-Tg level can effectively predict the efficacy of RAI, and elevated ps-Tg levels may indicate treatment failure. The results of this study were consistent with those studies. It was found that the lower the level of ps-Tg before RAI, the easier it was to obtain the best therapeutic response after the initial RAI, whether for PTC or PTMC. Ps-Tg was also an independent influencing factor of RAI efficacy in multivariate analysis. Through ROC curve analysis, the optimal cut-off value of ps-Tg was $10.61 \mathrm{ng} / \mathrm{mL}$ with the AUC of 0.806 in the PTC group, which was close to the analytical limit of Webb et al (ps-Tg< $10 \mathrm{ng} / \mathrm{mL}$ ). ${ }^{17}$ The optimal cut-off value of $\mathrm{ps}-\mathrm{Tg}$ was $5.87 \mathrm{ng} / \mathrm{mL}$ with the AUC of 0.690 in the PTMC group. The use of ps-Tg to predict the therapeutic effect of RAI has lower sensitivity in PTMC, which may be related to the lower ps-Tg of PTMC compared with PTC.

Liu et al believed that the tumor size was related to the aggressiveness and prognosis of papillary thyroid carcinoma, and that it could independently predict the treatment response of RAI. $^{18}$ In this study, because tumors were divided into PTMC and PTC groups according to the largest diameter of the tumor, there was no statistical difference in tumor size in the RAI treatment response of the PTMC and PTC groups. However, the overall RAI treatment response of the PTMC group was significantly better than that of the PTC group, which also reflects the correlation between tumor size and prognosis. After further dividing PTMC into two groups according to the maximum diameter: $>0.5 \mathrm{~cm}$ and $\leq 0.5 \mathrm{~cm}$, the group with $\leq 0.5 \mathrm{~cm}$ had better treatment response, which was consistent with the results reported by Lim et al. ${ }^{19}$ But it was excluded from the multivariate analysis, which means that the maximum diameter $\leq 0.5 \mathrm{~cm}$ was not an independent protective factor for PTMC RAI treatment response. Former studies have shown that PTMC was an early lesion of PTC, and no significant difference was found between them at the gene level. ${ }^{9}$ This study found that the proportion of ER gradually decreased with the maximum diameter of tumors from $\leq 0.5 \mathrm{~cm}, 0.5-1.0 \mathrm{~cm}$, $1.0-2.0 \mathrm{~cm}$ to $>2.0 \mathrm{~cm}$, which was consistent with the study by Liu et $\mathrm{al}^{18}$ which showed that the risk of NER increased with the increase in tumor diameter. It also showed that PTMC is the early stage of PTC. The guidelines proposed by the World Health Organization for early detection, early diagnosis, early treatment and improvement of cure rate of tumors also stated similar opinions. $^{20}$

In previous literature, ${ }^{12,18,21}$ the presence of ETE and HT was often mentioned as influencing factors for RAI treatment of postoperative DTC patients. There was no significant effect of ETE and HT on the efficacy of PTC and PTMC in this study, which may be related to the exclusion of distant metastasis and TgAb-positive patients. 


\section{Conclusion}

The efficacy of initial RAI in PTMC patients was significantly better than that in PTC patients. Gender, N stage, and ps-Tg were independent influencing factors of the initial RAI response of PTC and PTMC. There was no significant difference in the initial RAI efficacy between PTMC (male, N1b stage and ps-Tg> $5.87 \mathrm{ng} / \mathrm{mL}$ ) and PTC patients, suggesting the necessity of RAI in such PTMC patients. For patients with stage N1a PTMC, individualized treatment should be carried out to avoid excessive and insufficient diagnosis and treatment, so as to maximize the benefits for patients.

\section{Disclosure}

The authors report no conflicts of interest in this work.

\section{References}

1. Chinese Association of Thyroid Oncology. Chinese Expert Consensus on Diagnosis and Treatment of Papillary Thyroid Microcarcinoma. Chin J Clin Oncol. 2016;43(10):405-411. doi:10.3969/j.issn.10008179.2016.10.001

2. Chinese Society of Nuclear Medicine. Clinical guidelines for ${ }^{131}$ I therapy of differentiated thyroid cancer [J]. China J Nucl Med Mol Imaging. 2014;34(4):264-278.

3. Feng S, Tan J, et al. Clinical characteristics and 131I efficacy of papillary thyroid microcarcinoma and papillary thyroid non-micro carcinoma [J]. Int J Radiat Med Nucl Med. 2018;42:2. doi:10.3760/ cma.j.issn.1673-4114.2018.02.003

4. Haugen BR, Alexander EK, Bible KC, et al. 2015 American Thyroid Association management guidelines for adult patients with thyroid nodules and differentiated thyroid cancer: the American Thyroid Association guidelines task force on thyroid nodules and differentiated thyroid cancer[J]. Thyroid. 2016;26(1):1-133. doi:10.1089/ thy. 2015.0020

5. Domínguez JM, Nilo F, Martínez MT, et al. Papillary thyroid microcarcinoma: characteristics at presentation, and evaluation of clinical and histological features associated with a worse prognosis in a Latin American cohort [J]. Arch Endocrinol Metab. 2018;62(1):6-13. doi:10.20945/2359-3997000000013

6. Noguchi S, Yamashita H, Uchino S, et al. Papillary microcarcinoma[J]. World J Surg. 2008;32(5):747-753. doi:10.1007/ s00268-007-9453-0

7. Hay ID. Management of Patients with Low-Risk Papillary Thyroid Carcinoma [J]. Endocr Pract. 2007;13(5):521-533. doi:10.4158/ EP.13.5.521

8. M B Amin, Edge S, Greene F, et al. 2017 AJCC Cancer Staging Manual [M]. 8th. New York: Springer; 2017.
9. Youn Y-K, Kim H, Park W-Y, et al. Comparative analysis of gene expression profiles of papillary thyroid microcarcinoma and papillary thyroid carcinoma [J]. J Cancer Re Therapeutics. 2010;6(4):452-457. doi:10.4103/0973-1482.77103

10. Roh J-L, Kim J-M, Park CI. Central cervical nodal metastasis from papillary thyroid microcarcinoma: pattern and factors predictive of nodal metastasis [J]. Ann Surg Oncol. 2008;15(9):2482-2486. doi:10.1245/s10434-008-0044-6

11. Long Y, Caixia A, Zhang W. Characteristics of 185 thyroid micropapillary carcinoma patients undergoing 131I treatment [J]. China J Nucl Med Mol Imaging. 2019;39:9. doi:10.3760/cma.j.issn.20952848.2019.09.005

12. Li H, Zhang Y-Q, Wang C, et al. Delayed initial radioiodine therapy related to incomplete response in low- to intermediate-risk differentiated thyroid cancer. Clin Endocrinol (Oxf). 2018;88(4):601-606. doi:10.1111/cen.13551

13. Wang X, Song Q, Dongdong X. Effects of Timing of Initial Postoperative Radioactive Iodine Therapy on the Outcome of Patients with Differentiated Thyroid Cancer [J]. J China Med Univ. 2019;48(4):359-369. doi:10.12007/j.issn.0258-4646.2019.04.016

14. Xin L, Lin Y. Change of pre-ablative thyroid-stimulating hormone after thyroid hormone withdrawal and its response to ${ }^{131} \mathrm{I}$ therapy in patients with low to intermediate risk differentiated thyroid cancer [J]. China J Nucl Med Mol Imaging. 2016;35(6):389-393. doi:10.3760/cma.j.issn.2095-2848.2016.05.003

15. Gonzalez C, Aulinas A, Colom C, et al. Thyroglobulin as early prognostic marker to predict remission at 18-24 months in differentiated thyroid carcinoma. Clin Endocrinol (Oxf). 2014;80 (2):301-306. doi:10.1111/cen.12282

16. Kim H, Kim S-J, Kim I-J, et al. Limited clinical value of periablative changes of serum markers in the prediction of biochemical remission in patients with papillary thyroid cancer [J]. Nucl Med Mol Imaging. 2013;47(4):268-272. doi:10.1007/s13139-013-0220-x

17. Webb RC, Howard RS, Stojadinovic A, et al. The utility of serum thyroglobulin measurement at the time of remnant ablation for predicting disease-free status in patients with differentiated thyroid cancer: a meta-analysis involving 3947 patients. J Clin Endocrinol Metab. 2012;97(8):2754-2763. doi:10.1210/jc.2012-1533

18. Liu J, Liang J, Lin Y. Relationship between preablative stimulated thyroglobulin and the excellent response in differentiated thyroid carcinoma [J]. China Oncology. 2019;29(2):125-130. doi:10.19401/ j.cnki.1007-3639.2019.02.005

19. Lim YC, Choi EC, Yoon Y-H, et al. Central lymph node metastases in unilateral papillary thyroid microcarcinoma[J]. Br J Surg. 2009;96 (3):253-257. doi:10.1002/bjs.6484

20. WHO. Guide to Cancer Early Diagnosis. ISBN: 978-92-4-151194-0. World Health Organization; 2017.

21. $\mathrm{Du} \mathrm{F}, \mathrm{Hu} \mathrm{S}, \mathrm{Wu} \mathrm{C}$, et al. [Analysis of the factors affecting the efficacy of $\left({ }^{131}\right) \mathrm{I}$ remnant ablation in patients after thyroidectomy for papillary thyroid microcarcinoma].. Zhonghua Zhong Liu Za Zhi. 2018;40(8):610-613. doi:10.3760/cma.j.issn.0253-3766.2018.08.009

\section{Publish your work in this journal}

Cancer Management and Research is an international, peer-reviewed open access journal focusing on cancer research and the optimal use of preventative and integrated treatment interventions to achieve improved outcomes, enhanced survival and quality of life for the cancer patient.
The manuscript management system is completely online and includes a very quick and fair peer-review system, which is all easy to use. Visit http://www.dovepress.com/testimonials.php to read real quotes from published authors. 